\title{
Resonance Raman study of linear carbon chains formed by the heat treatment of double-wall carbon nanotubes
}

\author{
C. Fantini, ${ }^{1}$ E. Cruz, ${ }^{2}$ A. Jorio, ${ }^{1}$ M. Terrones, ${ }^{2}$ H. Terrones,${ }^{2}$ G. Van Lier, ${ }^{3}$ J-C Charlier, ${ }^{3}$ M. S. Dresselhaus, ${ }^{4}$ R. Saito, ${ }^{5}$ \\ Y. A. Kim, ${ }^{6}$ T. Hayashi, ${ }^{6}$ H. Muramatsu, ${ }^{6}$ M. Endo, ${ }^{6}$ and M. A. Pimenta ${ }^{1}$ \\ ${ }^{1}$ Departamento de Física, Universidade Federal de Minas Gerais, Belo Horizonte, MG, 30123-970, Brazil \\ ${ }^{2}$ Advanced Materials Department, IPICYT, San Luis Potosí, 78216, Mexico \\ ${ }^{3}$ Université Catholique de Louvain, PCPM \& CERMIN, B-1348 Louvain-la-Neuve, Belgium \\ ${ }^{4}$ Massachusetts Institute of Technology, Cambridge, Massachusetts 02139-4307, USA \\ ${ }^{5}$ Department of Physics, Tohoku University and CREST JST, Aoba Sendai 980-8578, Japan \\ ${ }^{6}$ Faculty of Engineering, Shinshu University 4-17-1 Wakasato, Nagano-shi 380-8553, Japan
}

(Received 30 March 2006; published 25 May 2006)

\begin{abstract}
We study the resonance behavior of the unusual Raman feature known as the coalescence-inducing mode (CIM), observed at $\sim 1850 \mathrm{~cm}^{-1}$, in samples of double-wall carbon nanotubes annealed at high temperatures. Resonance Raman spectra taken with different laser energies show that the intensity of the CIM band exhibits a maximum around $2.20 \mathrm{eV}$. By comparing the resonance Raman experimental results with first-principles calculations for the vibrational frequency and the energy gap, we propose that the CIM feature is associated with short carbon chains with an odd number of atoms, interconnecting the nanotube surfaces.
\end{abstract}

DOI: 10.1103/PhysRevB.73.193408

PACS number(s): 78.30.Na, 63.22.+m, 78.20.Bh, 78.66.Tr

The Raman spectra of carbon nanotubes exhibit weak features in the spectral range between 1600 and $2000 \mathrm{~cm}^{-1}$ that are ascribed to a second-order Raman process involving the combination of two phonons. ${ }^{1,2}$ However, the observation of unusual and strong spectral features around $1850 \mathrm{~cm}^{-1}$ have been reported recently in the Raman spectra of carbon nanotube systems, ${ }^{3-5}$ and have been ascribed to the vibration of one-dimensional (1D) chains of carbon atoms. In particular, a strong and sharp feature at $\sim 1850 \mathrm{~cm}^{-1}$ is observed in the Raman spectra of double-wall nanotube (DWNT) samples heat treated at high temperatures and, since it appears at specific annealing temperatures $\left(T_{\mathrm{htt}}\right)$ that occur just below the $T_{\mathrm{htt}}$ needed for full coalescence of DWNTs, it is named the coalescence-inducing mode (CIM).

To characterize this unusual Raman feature at $\sim 1850 \mathrm{~cm}^{-1}$, we report a complete resonance Raman study of this phonon band, using many different laser excitation energies $\left(E_{\text {laser }}\right)$. The intensity dependence of the CIM band on $E_{\text {laser }}$ is here determined, and is shown to exhibit a maximum at around $2.2 \mathrm{eV}$. The experimental results are compared with theoretical calculations, using either density functional theory (DFT) or Hartree-Fock (HF) approaches, for finite carbon chains containing a small number of carbon atoms for which the normal mode frequencies and energy gaps were obtained. According to the calculations presented here, the vibrational frequencies and the resonance behavior obtained experimentally are in close agreement with the formation of short linear carbon chains during the coalescence process, with the chains having an odd number of carbon atoms fixed to the carbon nanotube walls under different environments (deformed and twisted chains).

Room-temperature Raman spectra were recorded in the backscattering configuration using a Dilor XY triple monochromator, equipped with a cooled charge-coupled device (CCD) and using several different laser line excitations from an $\mathrm{Ar}-\mathrm{Kr}$ ion laser and a dye laser in the range 1.9-2.7 eV. A laser power $<1 \mathrm{~mW}$ was focused on a $\sim 2 \mu \mathrm{m}^{2}$ spot during the measurements.
The samples studied here consist of highly purified DWNT bundles synthesized by a catalytic chemical vapor deposition method. ${ }^{6,7}$ The diameter distribution of the sample is $0.77 \leq d_{t} \leq 0.90 \mathrm{~nm}$ for the inner tubes and $1.43 \leq d_{t}$ $\leq 1.60 \mathrm{~nm}$ for the outer tubes. ${ }^{6,7}$ Both undoped and B-doped DWNTs were investigated after heat treatment at $T_{\mathrm{htt}}$ between 1000 and $2000{ }^{\circ} \mathrm{C}$. The CIM feature reaches its maximum intensity for the undoped sample when $T_{\mathrm{htt}}=1500{ }^{\circ} \mathrm{C}$ and for the B-doped DWNTs when $T_{\mathrm{htt}}=1300{ }^{\circ} \mathrm{C}$.

In order to understand the Raman frequencies observed experimentally in our spectra, we performed theoretical calculations optimizing the geometry and obtaining their vibrational frequencies on different types of carbon chains (with fixed ends and with loose ends) using two methods: (1) Density functional theory within the local density approximation (LDA), by means of the ABINIT code, ${ }^{8}$ and (2) Hartree-Fock simulations using GAUSSIAN03 (Ref. 9) in conjunction with the $6-311++\mathrm{G}(d, p)$ basis that includes polarization and diffuse functions on all atoms. The studied systems consist of carbon chains, 3-10 atoms in length. For LDA calculations, the chains were placed in a supercell and the separation between chains in all directions was large enough to avoid interactions among them. Finite carbon chains with and without benzene rings at the ends were considered for the HF method.

We also performed molecular dynamics (MD) calculations for elucidating the coalescence mechanism of DWNTs at the atomic level using the atom centered density matrix propagation (ADMP) molecular dynamics model at the AM1 semiempirical level of theory, using GAUSSIAN03 (Ref. 9) The calculations were carried out on two adjacent $(5,5)$ carbon nanotubes, with $12 \mathrm{~B}$ atoms placed between two adjacent parallel tubes along their axes.

Raman measurements were performed on the undoped and boron-doped DWNT samples using several excitation laser energies. Figure 1(a) shows a plot of the Raman spectrum of the undoped sample for $T_{\mathrm{htt}}=1500{ }^{\circ} \mathrm{C}$, measured 

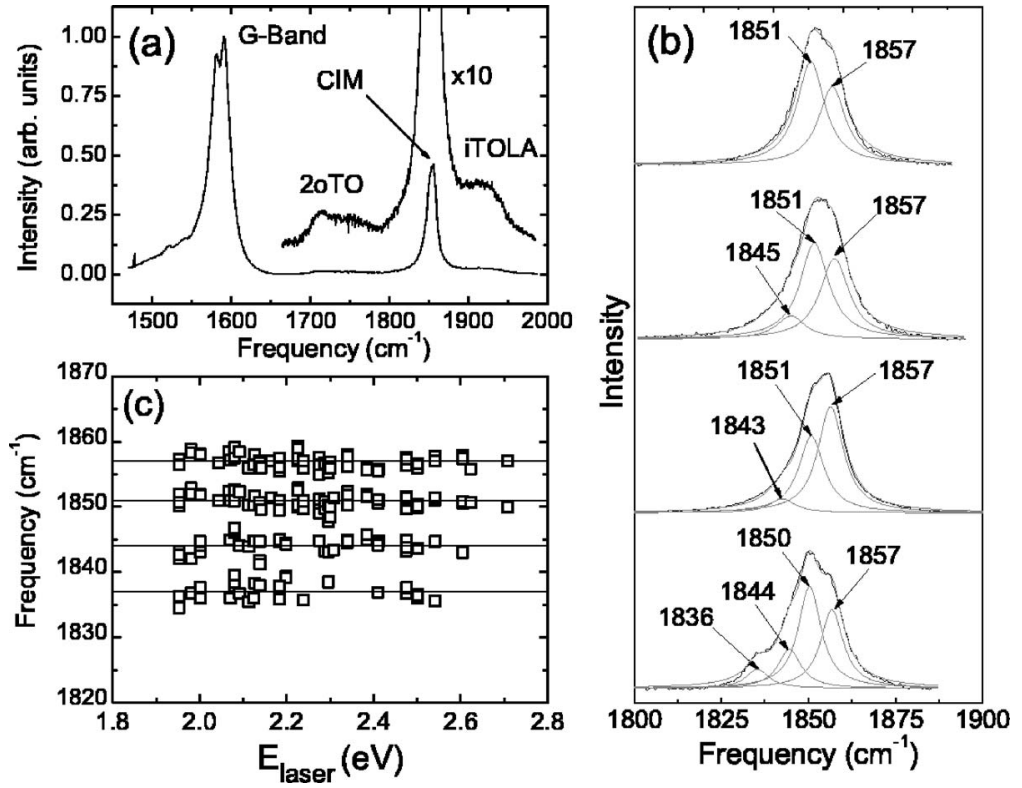

FIG. 1. (a) Raman spectrum of undoped DWNTs heat treated at $1500{ }^{\circ} \mathrm{C}$ where we can see the $G$ band, the CIM band, and the two combination modes 2oTO and iTOLA with the intensity multiplied by 10 . (b) First-order CIM bands obtained for $E_{\text {laser }}=2.20 \mathrm{eV}$ at different locations on an undoped sample heat treated at $1500{ }^{\circ} \mathrm{C}$. The spectra are fit with a sum of Lorentzians and their frequencies are displayed in $\mathrm{cm}^{-1}$. (c) Frequency vs $E_{\text {laser }}$ for all Lorentzian peaks used to fit the CIM feature. with $E_{\text {laser }}=2.20 \mathrm{eV}$ (where the CIM feature shows its maximum intensity). We can here observe the presence of the well-known graphitelike $G$ band whose intensity was normalized to exhibit the same value in all spectra. We observe also weak features at around 1700 and $1900 \mathrm{~cm}^{-1}$, that appear in the spectrum with the intensity multiplied by 10 , which are ascribed to the two phonon combination modes (2oTO and iTOLA) (Ref. 1), and the CIM band is observed strongly at around $\sim 1850 \mathrm{~cm}^{-1}$. Figure 1(b) shows the CIM Raman spectra obtained with $E_{\text {laser }}=2.20 \mathrm{eV}$, from four different sample locations of the laser spot on the undoped specimen $\left(T_{\mathrm{htt}}=1500{ }^{\circ} \mathrm{C}\right)$. We observed that the detailed shape and the mean frequency of the CIM band is somewhat dependent on the position of the laser spot on the sample. The CIM features obtained in all measured spectra were fitted by a sum of Lorentzian peaks, each one with a linewidth of $\sim 10 \mathrm{~cm}^{-1}$, also in agreement with the linewidth of the peaks previously observed in MWNT samples. ${ }^{3}$ The CIM feature in annealed DWNTs is clearly composed of more than one peak, and up to four peaks were necessary to fit all observed spectra [see Fig. 1(b)]. Four frequencies at $\sim 1837$, $\sim 1844$, $\sim 1851$, and $\sim 1857 \mathrm{~cm}^{-1}$, with a precision of $\pm 2 \mathrm{~cm}^{-1}$ could be clearly distinguished. The two highest frequency peaks are the most intense ones, and the peaks at $\sim 1844$ and $\sim 1837 \mathrm{~cm}^{-1}$ are less intense and sometimes absent from the spectra. The frequency values obtained from the analysis of all spectra, recorded with different laser excitation energies, are shown in Fig. 1(c) as a function of $E_{\text {laser }}$. The absence of a dispersive behavior demonstrates that these peaks are not related to the combination modes present in all graphitic materials, ${ }^{1}$ and reveals that the CIM band is associated with a unique form of carbon.

Since the intensities of the CIM peaks depend on the location of the laser spot on the sample, we have measured the Raman spectrum of the CIM feature at different locations on the sample (up to six locations) for each $E_{\text {laser }}$ between 1.9 and $2.7 \mathrm{eV}$, and subsequently, we considered an average value in order to obtain the CIM mean intensity for each CIM peak and each laser energy. Figure 2 shows the reso- nance Raman profile, i.e., the dependence of the integrated area of the CIM band as a function of $E_{\text {laser }}$ for the samples (a) without boron $\left(T_{\mathrm{htt}}=1500{ }^{\circ} \mathrm{C}\right)$ and (b) with boron doping $\left(T_{\mathrm{htt}}=1300{ }^{\circ} \mathrm{C}\right)$. All the data were normalized to keep the intensity of the $G$ band constant. Due to the large number of nanotube $(n, m)$ species in the DWNT sample, the $G$-band intensity is not expected to depend significantly on $E_{\text {laser }}$ and can be considered as a good internal parameter for normalization. Figure 2 shows that the general resonance behavior is quite similar for both the doped and undoped samples, indicating that B atoms do not play a role in the vibration that gives rise to the CIM band. We clearly observe three resonance maxima in the profile around 2.2, 2.4 and $2.6 \mathrm{eV}$ (see arrows in Fig. 2), for both doped and undoped samples.
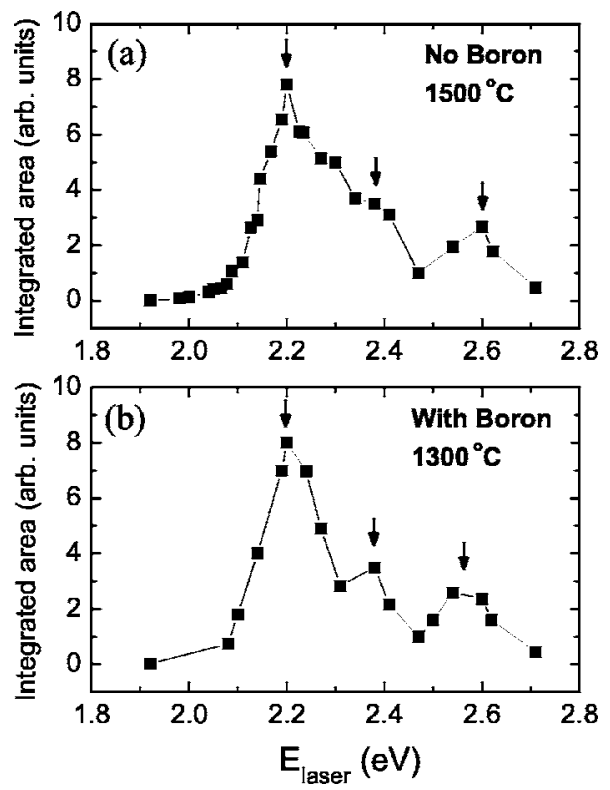

FIG. 2. Resonance Raman profile for the CIM feature of (a) the undoped and (b) the B-doped DWNT samples subjected to $1500^{\circ} \mathrm{C}$ and $1300{ }^{\circ} \mathrm{C}$ heat treatment, respectively. The arrows indicate the intensity maxima of the electronic resonance with $E_{\text {laser }}$. 
It is important to stress that the CIM feature composed of four peaks was observed for every laser excitation energy, including the 2.2-, 2.4- and 2.6-eV resonance maxima, their relative intensities always varying from location to location in the sample. Therefore, we could not relate any resonance maxima with a specific Raman peak in the CIM feature.

Zhao et al. ${ }^{3}$ observed sharp and intense Raman bands around 1825 and $1850 \mathrm{~cm}^{-1}$ in the Raman spectra of multiwall carbon nanotubes, and ascribed those bands to a long linear carbon chain, containing more than 100 atoms, and inserted inside a multiwall nanotube. Jinno et al. ${ }^{5}$ observed similar Raman peaks in MWNTs and DWNTs, ascribing these modes to linear carbon chains inside inner carbon nanotubes with small diameter $(\approx 0.6 \mathrm{~nm})$. The highfrequency band, around $1850 \mathrm{~cm}^{-1}$, is consistent with the CIM band reported in the present work [see Fig. 1(c)], but the sharp peak observed by Zhao et al. ${ }^{3}$ and Jinno et al. ${ }^{5}$ at $1825 \mathrm{~cm}^{-1}$ was not observed in our samples. The observation of different frequencies was ascribed to long linear carbon chains with different structures (cumulenic or polyynic). ${ }^{3}$ However, it is known that a linear chain with one atom per unit cell (cumulenic) does not have a Raman-active mode, as discussed recently by Rusznyák et al. ${ }^{10}$ Rusznyák et al. ${ }^{10}$ also show that the interaction of the inside carbon chains with the host carbon nanotube can suppress the Peierls distortion responsible for the formation of the polyynic structure. Furthermore, prior studies of long linear chains of $s p$-bonded carbon atoms, also known as carbynes, show that the $C \equiv C-C$ stretching mode frequency of a polyynic chain is about $2000 \mathrm{~cm}^{-1}$ (Ref. 11).

From our MD calculations, atomic carbon threads appear during nanotube coalescence. Besides the formation of carbon threads on the outer nanotube shell, MD simulations also show carbon chains containing 3,4 , and 5 atoms linking the inner and outer tubes covalently to the hexagonal network [see highlighted chain in Fig. 3(a)]. Another interesting and possible geometry is the interconnections established between the inner diameter tubes [e.g., binocularlike structures; see Fig. 3(b)].

Table I shows the calculated results for the vibrational normal mode frequencies and energy gaps of linear chains with a small number of carbon atoms and fixed ends. The frequencies of the stretching modes were calculated by first obtaining the second derivative of the total energy with respect to atomic displacements in the axial direction, and then by constructing and diagonalizing the dynamical matrix. Based on our MD calculations, we considered both fixed and unfixed carbon chains for calculating the Raman frequencies. The energy gaps for these 1D systems were calculated using the LDA approach.

One can notice that the energy gaps obtained (using the LDA approach) for the odd-numbered linear chains with fixed ends, and containing a small number of atoms, correspond to the range of electronic energies for which the CIM intensity is enhanced (e.g., $2 \mathrm{eV}$ ). We should emphasize that the energies obtained with the LDA method are underestimated and they are usually $10 \%$ lower than the actual value. Additional calculations using more sophisticated frameworks are underway. On the other hand, the energy gaps of fixed $\mathrm{C} 4$ and C6 chains are in the UV (Table I) region, with values
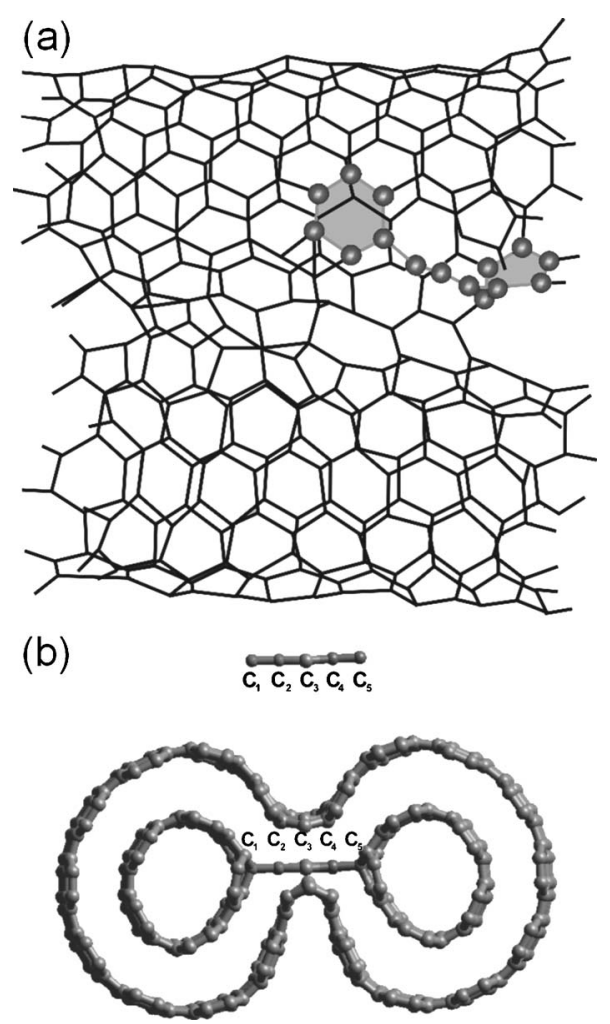

FIG. 3. (a) Molecular model obtained during MD calculations at $3000 \mathrm{~K}$, revealing the presence of carbon threads that form prior to the full coalescence of the nanotubes. The ends of each chain (highlighted in gray) are fixed to a hexagonal carbon network. (b) Molecular model considering an alternative framework where the C5 chain interconnects the inner nanotubes of two coalescing DWNTs.

much higher than the experimental laser energy values. This result leads us to conclude that the observed CIM feature is associated with the odd-numbered chains.

The calculated frequencies for the Raman-active stretching modes of the chains with 5 and 7 atoms, located at 1757 and $2098 \mathrm{~cm}^{-1}$, could be related to the experimental value of ca. $1850 \mathrm{~cm}^{-1}$ [see Table I and Fig. 1(a)]. We should note that the LDA approach also overestimates the Raman frequencies by 10\%-15\% (Ref. 12). Therefore, the Raman mode for the fixed linear C7 chain $\left(2098 \mathrm{~cm}^{-1}\right)$ would be in agreement with the experiment, since the calculated Raman mode for the fixed linear $\mathrm{C} 5$ chain $\left(1757 \mathrm{~cm}^{-1}\right)$ is below the

TABLE I. Vibrational stretching mode frequencies $\left(\omega_{\text {chain }}\right)$ and gap energy $\left(E_{\text {gap }}\right)$ for carbon chains with fixed ends, calculated for carbon chains containing 3-7 atoms. The Raman-active modes are boldface.

\begin{tabular}{lcc}
\hline \hline No. of atoms & $\begin{array}{c}\omega_{\text {chain }} \\
\left(\mathrm{cm}^{-1}\right)\end{array}$ & $\begin{array}{l}E_{\mathrm{Gap}} \\
(\mathrm{eV})\end{array}$ \\
\hline 3 & 1701 & 2.19 \\
4 & $1094, \mathbf{1 9 6 4}$ & 4.13 \\
5 & $935, \mathbf{1 7 5 7 , 2 1 7 7}$ & 1.98 \\
6 & $729, \mathbf{1 4 0 7}, \mathbf{1 9 0 6 , \mathbf { 2 0 5 6 }}$ & 3.02 \\
7 & $637, \mathbf{1 2 6 4}, 1756, \mathbf{2 0 9 8}, 2219$ & 1.68 \\
\hline \hline
\end{tabular}


experimental CIM band (ca. $1850 \mathrm{~cm}^{-1}$ ). However, when twisting this 5-atom chain, we could achieve upshifts of ca. $100-150 \mathrm{~cm}^{-1}$ that could fit the experiment (see below). The existence of a stable linear chain with more than eight atoms seems unlikely for DWNTs during coalescence. If we now consider unfixed linear carbon chains (or only fixed at one end), the active Raman frequencies (not shown here) are much higher than those for fixed systems at both ends. To the best of our knowledge, the idea of using fixed carbon chains has not been considered hitherto. So we conclude that C5 and $\mathrm{C} 7$ with fixed ends are the best candidates for the CIM.

We should also consider that, during DWNT coalescence, different types of highly compressed (even deformed and twisted) C5 chains are present. The existence of different compressed and deformed chains could explain the different maxima observed in the resonance behavior of the CIM intensity and the fact that the CIM band is composed of different Lorentzian peaks (Fig. 1). Furthermore, the Raman frequencies obtained using the HF approach, which considers fixed linear chains of 4 and 5 carbon atoms fixed to two benzene rings, provide additional valuable information. In particular, we found Raman-active frequencies for the central bonds of 2506 and $1820 \mathrm{~cm}^{-1}$, respectively. In order to evaluate the effect of curvature in these systems, the benzene molecules of the $\mathrm{C} 5$ chain were bent to coincide with the curvature of a $(10,10)$ carbon nanotube. The benzene molecules are connected with a C5 chain with the same bond lengths as the flat system, so no structural optimization was performed. Here, the Raman frequency is slightly higher, with a value of $1825 \mathrm{~cm}^{-1}$. This result suggests once more that the different peaks that compose the CIM band could be related to the bending of linear chains that are subjected to strains, deformations, and compression during coalescence.

In summary, we studied the line shape and the resonance behavior of the CIM Raman feature observed in heat-treated DWNT bundle samples. It was observed that the intensity of the CIM band depends strongly on the laser energy and exhibits a maximum value at $E_{\text {laser }}=2.2 \mathrm{eV}$. From the comparison between the experimental results with numerous theoretical calculations, we propose that the CIM feature observed at $\sim 1850 \mathrm{~cm}^{-1}$ is related to short and odd carbon chains (e.g., C5 or C7), that are deformed when linking adjacent DWNTs.

The Brazilian authors acknowledge financial support from the Instituto de Nanosciências - CNPq and CAPES. A.J. acknowledges V. Zólyomi and J. Kürti for important discussions. R.S. acknowledges X. Zhao for helpful discussions and a Grant-in-Aid (No. 16076201) from MEXT, Japan. This work was also sponsored by CONACYT-Mexico Grants No. 45762 (H.T.), 45772 (M.T.), 41464-Inter American Collaboration (M.T.), 42428-Inter American Collaboration (H.T.), the MIT-CONACYT program (M.S.D., M.T.), 2004-01-013/ SALUD-CONACYT (M.T.), and PUE-2004-CO2-9 Fondo Mixto de Puebla. M.S.D. acknowledges support from NSF Grant No. DMR 04-05534. J.-C.C. acknowledges the FNRS of Belgium for financial support. Part of this work is supported by the Belgian Program on Interuniversity Attraction Poles (PAI5/1/1), to the ARC sponsored by the Communauté Française de Belgique, NANOQUANTA and FAME Networks of Excellence.
${ }^{1}$ V. W. Brar, Ge. G. Samsonidze, M. S. Dresselhaus, G. Dresselhaus, R. Saito, A. K. Swan, M. S. Ünlü, B. B. Goldberg, A. G. Souza Filho, and A. Jorio, Phys. Rev. B 66, 155418 (2002).

${ }^{2}$ R. Saito, A. Jorio, A. G. Souza Filho, G. Dresselhaus, M. S. Dresselhaus, and M. A. Pimenta, Phys. Rev. Lett. 88, 027401 (2002).

${ }^{3}$ X. Zhao, Y. Ando, Y. Liu, M. Jinno, and T. Suzuki, Phys. Rev. Lett. 90, 187401 (2003).

${ }^{4}$ K. McGuire, N. Gothard, P. L. Gai, M. S. Dresselhaus, G. Sumanasekera, and A. M. Rao, Carbon 43, 219 (2005).

${ }^{5}$ M. Jinno, Y. Ando, S. Bandow, J. Fan, M. Yudasaka, and S. Iijima, Chem. Phys. Lett. 418, 109 (2006).

${ }^{6}$ M. Endo, H. Muramatsu, T. Hayashi, Y. A. Kim, M. Terrones, and M. S. Dresselhaus, Nature (London) 433, 476 (2005).

${ }^{7}$ M. Endo, H. Muramatsu, T. Hayashi, Y.-A. Kim, G. Van Lier,
J.-C. Charlier, H. Terrones, M. Terrones, and M. S. Dresselhaus, Nano Lett. 5, 1099 (2005).

${ }^{8}$ X. Gonze, J.-M. Beuken, R. Caracas, F. Detraux, M. Fuchs, G.-M. Rignanese, L. Sindic, M. Verstraete, G. Zerah, F. Jollet, M. Torrent, A. Roy, M. Mikami, Ph. Ghosez, J.-Y. Raty, and D. C. Allan, Comput. Mater. Sci. 25, 478 (2002).

${ }^{9}$ M. J. Frisch, G. W Trucks, H. B. Schlegel, G. E. Scuseria, M. A. Robb, J. R. Cheeseman, J. A. Montgomery Jr., T. Vreven, K. N. Kudin, J. C. Burant, and J. M. Millam, GAUSSIAN 03 REVISION B.05 (Gaussian, Inc., Pittsburgh PA, 2003).

${ }^{10}$ Á. Rusznyák, V. Zólyomi, J. Kürti, S. Yang, and M. Kertesz, Phys. Rev. B 72, 155420 (2005).

${ }^{11}$ J. Kürti, C. Magyar, A. Balázs, and P. Rajczy, Synth. Met. 71, 1865 (1995).

${ }^{12}$ O. Dubay and G. Kresse, Phys. Rev. B 67, 035401 (2003). 